\title{
Essai de généalogie en didactique des mathématiques
}

\section{Claire Margolinas}

Le présent article cherche à donner des clés permettant de mieux comprendre la généalogie du champ de recherche en didactique des mathématiques en France. Je considère tout d'abord les ruptures qui ont fondé ce champ de recherche, en montrant les différences et les similarités avec celles qui sont à l'origine d'autres didactiques disciplinaires. Je montre ensuite les spécificités des théories fondatrices de la didactique des mathématiques: la théorie des situations didactiques, la théorie des champs conceptuels, la théorie anthropologique du didactique. J'aborde enfin une période plus récente du développement que marquent la prise en compte des classes ordinaires et la modélisation du rôle du professeur. En conclusion, je montre comment, partie d'une spécificité disciplinaire quasi-étroite, la didactique des mathématiques se retrouve maintenant partie prenante d'interactions fortes avec d'autres didactiques disciplinaires et d'autres champs des sciences de l'éducation

\section{Introduction}

Pour attaquer le travail qui m’a été demandé ici: faire un bilan et en même temps montrer certaines perspectives actuelles du champ et de la recherche en didactique des mathématiques, j'ai commencé par étudier les travaux à l'origine de cette demande (voir Sieber et Thévenaz, dans ce volume). Si l'histoire de la didactique du français y est évoquée, celle de la didactique des mathématiques est mal connue, sans doute parce que les membres de ma communauté de recherche ne se sont pas donné la peine d'écrire sur ce sujet dans des revues accessibles à un large public.

Le présent article essaye donc de donner des clés permettant de mieux comprendre la généalogie du champ de recherche en didactique des mathématiques en France. Je m'appuierai tout d'abord sur les ruptures qui ont fondé ce champ de recherche, en montrant les différences et les similarités avec celles qui sont à l'origine d'autres didactiques disciplinaires. J'exposerai ensuite les spécificités des théories fondatrices de la didactique des mathématiques: la théorie des situations 
didactiques, la théorie des champs conceptuels, la théorie anthropologique du didactique. Cela nous permettra enfin d'aborder une période plus récente du développement que marquent la prise en compte des classes ordinaires et la modélisation du rôle du professeur. En conclusion, je présenterai comment, partie d'une spécificité disciplinaire quasi-étroite, la didactique des mathématiques se retrouve maintenant partie prenante d'interactions fortes avec d'autres didactiques disciplinaires et d'autres champs des sciences de l'éducation.

\section{Des ruptures fondatrices}

La constitution d'un nouveau champ scientifique est une chose assez rare, qui ne peut exister sans une impérieuse nécessité. La didactique des mathématiques française et, plus largement, internationale $s^{\prime}$ institue $^{1}$ dans les années 19501960, dans le cadre d'une histoire des mathématiques et de leur enseignement en plein bouleversement. En France, la réforme des programmes officiels dites des mathématiques modernes (entrée en vigueur en 1970) prend appui d'une part sur la restructuration des mathématiques et le travail du groupe Bourbaki (auquel Gustave Choquet appartient, fondateur de la Commission internationale pour l'étude et l'amélioration de l'enseignement en mathématiques CIEAEM), et d'autre part sur la psychologie et les travaux de Jean Piaget (également fondateur de la CIEAEM). En 1969, la naissance de l'Institut pour la Recherche et l'Enseignement des Mathématiques (IREM), qui avait pour rôle d'accompagner la réforme en permettant une interaction entre des professeurs de mathématiques de l'enseignement secondaire et des universitaires, a favorisé le rapprochement entre des problématiques de recherche et des problèmes d'enseignement.

L'échec de la réforme a été retentissant. Sa remise en cause, rendue publique dès 1973 par l'Association des Professeurs de Mathématiques de l'Enseignement Public (APMEP.), a été le point de départ de réformes successives qui ont contribué à effacer le changement profond provoqué par les mathématiques modernes. La didactique des mathématiques a donc connu, en France, un contexte d'émergence très singulier et une partie de ses singularités en portent sans doute la marque.

\section{Rupture avec L'applicationnisme}

Comme la didactique du français (Bronckart, 2001), la didactique des mathématiques s'inscrit en rupture avec l'application directe en classe de conceptions issues de cadres théoriques indépendants des phénomènes spécifiques liés à la transmission des connaissances mathématiques.

La psychologie et la réflexion épistémologique sur les mathématiques ont été à la source du mouvement des mathématiques modernes. Les liens entre la didactique des mathématiques, la psychologie et les mathématiques sont à la fois très étroits et toujours en question. La nécessité de réinterroger les disciplines qui 
contribuent à la réflexion sur l'enseignement et l'apprentissage des mathématiques s'est ainsi fait jour très tôt dans l'histoire de la didactique des mathématiques.

\section{Rupture avec l'innovation}

Cette deuxième rupture est une particularité de la didactique des mathématiques française. Il s'agit d'un aspect à la fois singulier et fondateur de ce champ.

L'échec de la réforme a montré dès les années 1970 que d'autres déterminants étaient à l'œuvre dans l'enseignement des mathématiques que le cognitif et les mathématiques en tant qu'entités séparées. Mais il a également rendu très prudents les didacticiens des mathématiques français quant à l'application rapide dans les classes et l'intervention directe sur le système d'enseignement.

La rupture avec l'innovation ne s'est pas accompagnée d'un abandon de la recherche sur le terrain, mais le rapport entre théorie et pratique est d'une autre nature, comme nous le verrons dans la deuxième partie de cet article.

\section{Nécessité d'une théorisation propre}

Une des originalités du paradigme français de recherche en didactique des mathématiques est de prendre au sérieux la recherche fondamentale, et non directement la réussite des élèves. Il s'agit de rechercher des conditions qui permettent en théorie de faire évoluer les connaissances des élèves et non pas seulement qui améliorent de fait l'enseignement: «Si une didactique scientifique existe, il faudra qu'elle permette de déduire les mesures méthodologiques les plus aptes à provoquer les acquisitions, d'une connaissance scientifique des processus de formation intellectuelle.» (Brousseau, 1975, cité par Perrin-Glorian, 1994, p. 101).

Brousseau considère, dès les années 1970, la didactique des mathématiques comme une science expérimentale, dans laquelle les résultats techniques sont envisagés comme des conséquences des résultats fondamentaux. Même si l'ambition d'amélioration de l'enseignement des mathématiques est présente dans les intentions, l'existence et la légitimité d'une recherche fondamentale sont posées.

\section{Théories et ingénieries}

La didactique des mathématiques française a produit de nombreux concepts et éléments théoriques. Parmi ceux-ci, trois théories jouent un rôle fondateur: la théorie des situations didactiques, dont le fondateur est Guy Brousseau; la théorie des champs conceptuels, développée par Gérard Vergnaud; la théorie anthropologique du didactique, élaborée par Yves Chevallard.

\section{La théorie des situations}

Les éléments premiers de la théorie des situations didactiques (Brousseau, 1972 pour un texte fondateur; Brousseau, 1998 pour une compilation d'articles 1970- 
1990) partent d'une reconstruction de l'épistémologie des mathématiques et de ses rapports avec l'enseignement, qui a été nommée épistémologie expérimentale à ses débuts.

Dans cette reconstruction, la différence entre savoir et connaissance est un des éléments clé. Le savoir est une construction sociale, qui résulte d'un processus historique. La connaissance est un acquis personnel, signe de l'équilibre entre un sujet et son milieu.

Le lien entre connaissance et savoir existe dans l'origine du savoir: une connaissance se transforme en construit social au fil d'une longue et hasardeuse élaboration. Le problème qui se pose est d'une part de comprendre les conditions d'émergence d'une connaissance et du savoir qui en résulte, d'autre part d'épurer ces conditions de manière à en comprendre, non pas les composantes factuelles (ce qui relève de l'histoire de la discipline) mais les éléments nécessaires, qui fondent la raison d'une connaissance: c'est la problématique des situations (non didactiques) fondamentales (Brousseau, 1986, repris dans Brousseau, 1998).

Le caractère non didactique doit être précisé: seule une connaissance utile peut être transformée en savoir (Conne, 1992). Cette utilité se révèle dans des situations qui ne sont pas construites pour enseigner, mais qui existent dans la vie courante, professionnelle, mathématique, etc. Le problème didactique qui se pose est alors de produire les conditions d'utilité de la connaissance dans le cadre spécifique d'une institution didactique donnée. Le résultat de cette construction s'appelle situation adidactique. Elle est conçue comme le coeur d'une situation didactique et vise à reconstituer artificiellement l'interaction fondamentale sujetmilieu. La qualité de la situation adidactique, c'est-à-dire ses propriétés pour l'acquisition du savoir visé n'est pas purement théorique, elle passe par une expérimentation.

La démarche de Guy Brousseau et de ses collaborateurs (nombreuses thèses soutenues sous sa direction depuis 1975) s'est appuyée par un dispositif expérimental très original: le Centre pour l'Observation et la Recherche sur l'Enseignement des Mathématiques ${ }^{2}$ (COREM), installé dans une école élémentaire de la région bordelaise. Cette école n'a été conçue ni comme une école pilote ni comme une école d'application: il ne s'agissait pas d'y mettre des méthodes à l'essai (Brousseau, 1998, p. 359), mais de permettre l'expérimentation et l'observation dans des conditions adéquates.

Nous pouvons revenir maintenant à la question de l'innovation. Le travail expérimental ainsi conçu relève de l'ingénierie (Artigue, 1988): il s'agit d'éprouver la théorie et non pas d'essayer des méthodes. Bien sûr, dès que des professeurs, des élèves, des parents, sont impliqués dans un tel processus, ce n'est pas si simple! Mais cette position est cohérente avec le fait que les ingénieries développées au COREM n'ont été publiées que de façon tout à fait confidentielle, dans des documents de littérature grise édités par l'IREM de Bordeaux et ont très rarement été diffusées au grand public (voir néanmoins Briand, Loubet \& Salin, 2004, 
pour une exception récente, qui marque peut-être le signe d'un changement).

Ce que montre ce travail expérimental, c'est que dans ces conditions, les situations construites permettent l'enseignement de certains savoirs et que les élèves y acquièrent des connaissances. Les conditions expérimentales ne sont jamais oubliées; il ne s'agit pas de faire croire que les mathématiques sont enseignables de cette manière dans toutes les écoles et par tous les professeurs.

Le travail de Brousseau se situe dans le cadre des mathématiques, même si de nombreux concepts produits dans la théorie ont a priori une portée générale. Certains ont migré dans d'autres didactiques, notamment en didactique de l'Education Physique et Sportive (Loquet, Garnier \& Amade-Escot, 2002). Brousseau ne conçoit pas cette extension à l'intérieur de la théorie: il est possible de se saisir de la théorie des situations pour autre chose que ce pour quoi elle a été conçue, mais cette possibilité est en quelque sorte sans garantie, sous la responsabilité des chercheurs et non sous le contrôle de la théorie elle-même.

\section{La théorie des champs conceptuels}

Gérard Vergnaud, psychologue, va développer son travail en cohérence avec les travaux piagétiens sur le plan des concepts, mais dans une perspective qui va, au départ, s'inscrire dans les recherches sur l'apprentissage des mathématiques (Vergnaud, 1981).

Comme Brousseau, Vergnaud a donc avant tout une visée théorique. Sa perspective est différente car elle s'inscrit à l'intérieur de la psychologie, dans laquelle elle prend ses racines. Pourtant, Vergnaud n'entretient pas avec la didactique des mathématiques une relation d'extériorité, il a acquis en quelque sorte la double nationalité. La communauté de didactique des mathématiques le reconnaît d'ailleurs comme tel puisque le colloque intitulé «Vingt ans de didactique des mathématiques en France» (Artigue, Gras, Laborde \& Tavignot, 1994) avait pour but un «hommage à Guy Brousseau et Gérard Vergnaud».

Vergnaud va s'intéresser aux relations entre la psychologie et la didactique, dans une perspective qui, nous le verrons, le démarque de l'applicationnisme. Il s'intéresse en effet à la «question des contenus d'enseignement à l'intérieur d'une psychologie du développement cognitif» (Brun, 1994, p. 71, c’est moi qui souligne).

[La] principale finalité [de la théorie des champs conceptuels] est de fournir un cadre qui permette de comprendre les filiations et les ruptures entre connaissances, chez les enfants et les adolescents, en entendant par 'connaissances' aussi bien les savoirs-faire que les savoirs exprimés. (Vergnaud, 1991, p. 135)

La question fondamentale à laquelle Vergnaud s'attaque est la même que celle qui intéresse Brousseau: celle des relations entre connaissance et savoir. L'instrument théorique qu'il se donne pour aborder cette question est celle de champ conceptuel. Il s'agit d'un concept dont la visée n'est pas celle de la construction ou de l'analyse micro-didactique d'une situation, mais celle de la prise en compte d'un ensemble plus large, centrée sur un concept, que l'on pourrait qualifier de meso-didactique (Margolinas, 1992a). 
Le champ conceptuel se caractérise par une double entrée:

La première entrée est celle des situations «[qui] n'a pas ici le sens de situation didactique mais plutôt celui de tâche» (Brun, 1994, p.146) et des schèmes "totalité dynamique organisatrice de l'action du sujet pour une classe de situations spécifiée» (p.14). Comme le souligne Brun, les schèmes «sont des organisations, produits de l'activité cognitive, et des organisateurs, instruments d'assimilation» (p. 73), ces deux aspects sont valorisés dans le champ conceptuel, qui cherche à conjuguer l'activité cognitive et le savoir mathématique. "Avec les invariants opératoires on cherche à donner un contenu mathématique aux organisations des conduites repérables en situation.» (p. 73).

La seconde entrée est celle des concepts et des théorèmes mathématiques. «Le champ conceptuel des structures additives est à la fois l'ensemble des situations dont le traitement implique une ou plusieurs additions ou soustractions, et l'ensemble des concepts et théorèmes qui permettent d'analyser ces situations comme des tâches mathématiques» (Vergnaud, 1991, p. 147).

Constituer un champ conceptuel demande de classer les situations, en considérant le sens des situations et des symboles. «La clef est de considérer l'action du sujet en situation, et l'organisation de sa conduite.» (p. 167). Le champ conceptuel s'organise "[en recherchant] les parentés et les ruptures à l'intérieur d'un ensemble de situations organisé par des idées elles-mêmes parentes, dans lesquelles les procédures, les représentations et les formulations puissent raisonnablement dériver les unes des autres» (p. 167).

Quelle est la position de Vergnaud par rapport à l'innovation ? Il a participé à de nombreuses ingénieries didactiques sur le long terme (par exemple, concernant le volume, voir l'ensemble des articles du volume 4 (1) de la revue Recherches en didactique des mathématiques, 1984). Par ailleurs, le travail de Gérard Vergnaud, en ce qui concerne principalement le champ conceptuel des structures additives, a été largement diffusé par les formateurs de mathématiques auprès des professeurs des écoles. Vergnaud a lui-même dirigé une collection de manuels de mathématiques pour l'école primaire ${ }^{3}$.

En ce qui concerne la relation de la théorie des champs conceptuels avec les mathématiques, la position de Vergnaud est celle du champ de la psychologie, qui n'a pas vocation à restreindre sa visée à un champ disciplinaire particulier. Il va ainsi se placer non seulement dans le cadre de la didactique des mathématiques, mais aussi dans la perspective des didactiques (Vergnaud, 1994a) puis de la didactique professionnelle (Vergnaud, 2005). Mais il conserve, quel que soit le champ, un ancrage dans les savoirs - qu'ils soient constitués dans la culture des disciplines ou dans la culture professionnelle - qui caractérise son approche.

\section{La théorie anthropologique du didactique}

Le travail d'Yves Chevallard, dont les premiers écrits de didactique des mathématiques sont publiés au début des années 80 , se situe d'emblée dans le cadre très large de l'anthropologie et assume la portée générique de sa théorisation. Dans 
un article fondateur de cette approche (Chevallard, 19924), il situe la didactique des mathématiques à l'intérieur de champs qui vont du général au spécifique. Il part du cadre anthropologique, dans lequel les termes primitifs sont les objets, les personnes, les institutions ${ }^{5}$. Il introduit ensuite la connaissance: connaittre un objet c'est avoir un rapport à cet objet; s'intéresser à ces rapports c'est faire de l'anthropologie de la connaissance ou de l'anthropologie cognitive.

Les institutions ne sont pas des espaces homogènes, il existe différentes positions en leur sein. En particulier, un sujet est en position d'élève au sein d'une institution donnée s'il existe des objets de l'institution, enjeux didactiques pour les sujets en position d'élève, tels que l'institution manifeste l'intention de rendre les rapports personnels du sujet à ces objets conformes aux rapports institutionnels à ces mêmes objets. C'est dans le cadre de cette étude que l'on peut parler d'anthropologie didactique de la connaissance, que l'on peut aussi appeler didactique de la connaissance ou didactique cognitive.

Chevallard introduit ensuite comme un terme primitif l'objet savoir: objets qui peuvent être appris et peuvent être enseignés et ne peuvent être connus sans être appris. Ainsi, l'anthropologie des savoirs est un sous domaine de l'anthropologie de la connaissance. Comme précédemment, on peut alors définir l'anthropologie didactique des savoirs ou la didactique des savoirs ou tout simplement la didactique. Comme il existe des sous domaines des savoirs - les mathématiques, la physique, etc. - il existe ainsi la didactique des mathématiques, la didactique de la physique, etc.

Chevallard assume ainsi pleinement l'insertion de la didactique des mathématiques à l'intérieur d'un domaine beaucoup plus vaste. Il prend le soin de situer les concepts introduits dans les cadres du plus général - l'anthropologie - au plus spécifique - la didactique d'un sous domaine du savoir.

Il partage avec Brousseau et Vergnaud l'ambition théorique et la nécessité de pointer la différence entre le travail de la théorie en tant que machine à produire des connaissances et le système modélisé. Il partage également la nécessité de prendre en compte les conditions concrètes de l'activité de l'élève. Le rapport au réel ne peut être un rapport innovant ou directement descriptif, c'est la théorie qui lui donne sens.

A ce stade de la théorisation, la théorie des situations didactiques apparaît à Chevallard comme complétant la théorie anthropologique du didactique. Voyons plus précisément comment Chevallard (1992) situe les différences entre ces deux approches:

[La théorie des situations] tend à privilégier le point de vue de l'économie et à laisser un peu en retrait le point de vue de l'écologie des systèmes. Ou, pour le dire plus concrètement, elle tend à se centrer sur le fonctionnement de la machine, en laissant un peu de côté l'étude des conditions de possibilité de ce fonctionnement. [...] Ou encore, pour avancer une autre formule, plus personnalisée mais au demeurant tout aussi approximative que les précédentes: Guy Brousseau me paraît «obsédé" par les conditions du bon fonctionnement des systèmes didactiques; je suis, 
quant moi, davantage fasciné par l'étude des conditions de possibilité de leur fonctionnement tout court - bon ou moins bon.» (p.103).

Si nous examinons maintenant les rapports du travail de Chevallard avec l'expérimentation ou l'observation dans le cadre scolaire, on va voir que l'écart avec l'innovation est ici maximum. D'une part, comme il le dit dans la citation précédente, il s'intéresse plus aux conditions de possibilité qu'aux transformations du système. D'autre part, il est plus centré, en tout cas dans la période 80-90, sur des phénomènes macro didactiques (comme la transposition didactique: Chevallard, 1985). Il existe pourtant une part cachée de ce travail théorique qui, comme chez Brousseau, passe par de très nombreuses ingénieries réalisées dans des classes de collège publiées avec ses collaborateurs à l'IREM de Marseille. Comme Brousseau, il considère comme inopportun de délivrer au professeur des séances toutes faites. Il cherche à favoriser de la part du professeur une réflexion d'un tout autre niveau de généralité qui permette de situer l'objet à enseigner dans un entendement plus général (comme il le formalisera plus tard: voir Chevallard, 2002a et 2002b). C'est dans cet esprit qu'il publie (en espagnol), un livre destiné au grand public (Chevallard, Bosch \& Gascón, 1996), consacré à l'étude des mathématiques, chaînon manquant entre l'enseignement et l'apprentissage.

\section{La rencontre des classes ordinaires}

Dans les années 90, un mouvement international de toutes les recherches en didactique des mathématiques ${ }^{6}$ pousse les chercheurs vers l'étude des classes ordinaires, c'est-à-dire dans lesquelles les séances n'ont pas fait l'objet d'un travail préalable entre le chercheur et le professeur. Ce mouvement est concomitant de celui d'une centration vers l'étude du professeur de la part de toute la communauté de didactique des mathématiques (voir notamment Brousseau, 1995; Chevallard, 1997, 2002a et 2002b; Margolinas, 1992a, 1992b, 2000, 2002; Vergnaud, 1994b, pour se limiter aux auteurs déjà cités dans cet article).

\section{Le travail du professeur}

Dans les travaux des années 70-80, le professeur a en effet été mis en quelque sorte entre parenthèses. Les expérimentations liées aux ingénieries de la théorie des situations didactiques s'intéressent au noyau dur des interactions élève/milieu et savoir/connaissance. Pour observer (indirectement) ces interactions, il est essentiel d'un point de vue méthodologique d'observer l'élève dans un cadre qui puisse être «le moins troublé possible» par le professeur et son projet social, dans lequel le professeur n'interviendra que comme organisateur des relations au milieu. Quant à elle, la théorie anthropologie du didactique ne décrit pas dans un premier temps la spécificité de la position du professeur, les travaux des années 80 concernant la transposition didactique n'abordent pas souvent la question du savoir effectivement enseigné, s'arrêtant souvent au savoir à enseigner. 
Dans le cas des ingénieries, même si le plus souvent le professeur participe aux recherches et à la mise en place des situations, le chercheur assume une partie du rôle du professeur, puisqu'il est le maître d'œuvre du processus à réaliser (et parfois le remplace même comme acteur en classe). Mercier (1998) a pointé la difficulté dans laquelle se sont longtemps trouvés les chercheurs en didactique visà-vis du professeur, en assumant une partie de son rôle:

En proposant une ingénierie d'aide à l'enseignement en échange de l'observation, le didacticien avait pris à sa charge une part de la responsabilité d'enseigner $d u$ professeur: au point de ne plus être vraiment en position d'observateur distancié, puisqu'il s'engageait dans un travail phénoménotechnique. (p. 292)

Ce modèle rend impossible la conception d'un rôle du professeur, car l'interaction entre chercheur et professeur est faussée: "L'identification des situations adidactiques est garantie par l'analyse a priori d'une ingénierie, les comportements enseignants observés sont alors des 'ratés': les 'effets de contrat' en sont alors une description suffisante.» (p. 295).

C'est parce que le professeur est parfois gênant que les chercheurs vont commencer à s'intéresser à lui au début des années 1990. Il est gênant parce qu'il n'assume pas son rôle comme le chercheur l'a conçu dans l'ingénierie, ou bien parce qu'il transforme d'une façon imprévue le savoir à enseigner. Relativement aux savoirs, il faut donc étudier non seulement la situation de l'élève mais aussi celle du professeur.

\section{Le rôle du professeur dans la dévolution des situations}

Le rôle du professeur dans la dévolution des situations (Margolinas, 2005) va me servir à montrer sur la base d'un exemple les évolutions de la didactique des mathématiques dans les années 1990.

Il convient tout d'abord de préciser le sens de ce terme dans le cadre de la théorie de situations didactiques, dans laquelle il apparaît en même temps que celui de situation adidactique:

Il ne suffit pas de "communiquer» un problème à un élève pour que ce problème devienne son problème et qu'il se sente seul responsable de le résoudre. Il ne suffit pas non plus que l'élève accepte cette responsabilité pour que le problème qu'il résout soit un problème "universel» dégagé de présupposés subjectifs.

Nous appelons "dévolution" l'activité par laquelle le professeur cherche à atteindre ces deux résultats. (Brousseau, 1986, p. 39)

Ce que le maître doit réaliser est donc une activité spécifiée uniquement par sa finalité.

Dans un premier temps (avant 1989), une vision naïve du rôle du professeur a souvent prévalu, plus ou moins explicitement (Margolinas, 1999). Le professeur y est vu comme ayant tout pouvoir pour installer une bonne situation, à partir du moment où ses convictions (ses représentations, son épistémologie, etc.) sont conformes au projet du chercheur. C'est ainsi que l'on s'est parfois mépris sur le rôle du professeur dans la dévolution des phases adidactiques, qui a parfois été 
envisagé comme suit (à tort, bien entendu, voir Margolinas, 1993, p. 35): 1. Le maître serait tout d'abord actif, il parlerait à la classe et présenterait le problème. Ce serait la phase de dévolution; 2. Le maître ne dirait plus rien, le problème étant devenu celui des élèves. Ce serait la phase adidactique, quasi-isolée $d u$ maître; 3. Le maître interviendrait à nouveau activement pour institutionnaliser le savoir. Ce serait la phase d'institutionnalisation. Si je m'y oppose, c'est parce que cette description n'est didactiquement pas viable et que le rôle du professeur y est envisagé sans tenir compte de la situation qui s'impose non seulement aux élèves mais également au professeur.

Dans un deuxième temps (durant les années 90), de nombreux travaux ont contribué au contraire à caractériser ce que dit et ce que fait le professeur pendant la dévolution d'une phase adidactique. Ils ont permis de disposer d'une description modélisée de l'activité du professeur (voir les travaux donnés en référence dans l'introduction de cette partie). Ces modèles ont permis de mieux comprendre les contraintes et les ressources de l'activité du professeur, c'est-à-dire de mieux comprendre la situation du professeur. Ils ont permis par exemple de comprendre que le maître ne pouvait pas se taire, même pendant les phases adidactiques (ce que les vidéos de classe, notamment celles du COREM, nous montraient d'ailleurs depuis longtemps), qu'il devait remplir une partie de son rôle et notamment celui de maintenir l'engagement des élèves, ce que Brousseau (1981) avait déjà partiellement décrit dans la gestion affective de la dévolution. Ils ont permis aussi de comprendre que l'élève n'est pas le seul à être contraint par le problème posé et que le professeur l'est aussi (Margolinas, 1992b).

Dans une période plus récente (fin 90, début 2000), de nombreuses recherches ont cherché à caractériser les situations effectives que le professeur installe, qu'il s'agisse d'une analyse locale, centrée sur une ou quelques séances (comme dans Margolinas, 2000) ou d'une analyse plus globale qui tient compte de l'ensemble d'un curriculum (comme dans Bosch, Espinoza \& Gascón, 2003; Roditi, 2005).

Donnons un exemple centré sur l'analyse locale, qui m'est plus familière. Quand il choisit, par exemple, un problème à poser aux élèves, le professeur ne peut en analyser toutes les caractéristiques. Il ne contrôle pas non plus les modifications parfois profondes que ses interventions font subir à la situation des élèves (voir Arsac \& Mante, 1988, le texte fondateur qui décrit ce problème). Le professeur installe ainsi, de fait, une situation, mais ne connaît pas a priori la façon dont les élèves peuvent investir cette situation. Or la plupart des situations peuvent être investies de façon différente par les élèves, non seulement du point de vue pédagogique (affectif, par exemple) mais aussi du point de vue didactique, c'est-à-dire du point de vue des savoirs en jeu dans la situation. Ainsi, là où le professeur souhaite inviter l'élève à travailler sur des nombres, celui-ci peut travailler sur des écritures (Comiti, Grenier \& Margolinas, 1995), ou bien le professeur peut engager la classe à faire de la numération alors que certains élèves persistent à interpréter la situation du point de vue spatial (Margolinas \& Tho- 
mazet, 2004). Dans un premier temps, les bifurcations didactiques (Margolinas, 2004) qui apparaissent ainsi ont été interprétées comme des dysfonctionnements de la situation didactique, comme des maladresses du professeur, dues à sa condition de travailleur (le professeur n'a en effet pas la possibilité d'analyser de façon fine tous les problèmes qu'il propose en classe), ce qui permet le rapprochement avec la question des malentendus, soulevés par les sociologues de l'éducation (Bautier \& Rochex, 2004).

Des travaux plus récents commencent à mettre en évidence que ce qui peut être vu par le didacticien comme un dysfonctionnement relève peut-être d'un fonctionnement dans la situation d'ensemble du professeur, qui ne repose pas exclusivement sur des déterminations didactiques (Goigoux, sous presse; Margolinas, 2002 et 2004; Robert, 2001; Rogalski, 2003). Ainsi, il peut être efficace pour le professeur de réunir des élèves autour d'un même problème, en faisant comme si ils étaient investis dans la même situation. L'abandon de l'établissement d'une situation pour tous est alors la négation de la dévolution, dans le sens propre du mot, au profit de l'engagement des élèves dans une tâche ayant des caractéristiques communes. Les intentions non didactiques du professeur sont à prendre en compte pour l'analyse didactique de la situation effective.

Tout se complique! La théorie des situations didactiques, dont la logique première est celle d'une construction a priori des situations expérimentées dans une ingénierie, devient la source d'outils théoriques pour l'analyse a posteriori des situations et révèle sa pertinence dans l'analyse de séquences d'enseignement d'autres disciplines que les mathématiques (Margolinas \& Goigoux, sous presse). De même, la théorie anthropologique du didactique se transforme en prenant en compte le travail du professeur et l'étude nécessaire de la praxéologie didactique et de ses relations fort complexes avec la praxéologie mathématique (Bosch, Espinoza \& Gascón, 2003; Bosch \& Gascón, 2002).

L'étude du travail du professeur conduit ainsi nécessairement à la prise en compte de déterminations qui ne sont pas d'origine didactique et qui pourtant interagissent de façon très étroite avec le didactique et ont des conséquences profondes sur les situations didactiques effectives. Ainsi, il n'est plus possible, si l'on prend en compte le professeur, de situer la théorisation didactique uniquement dans le cadre de la didactique d'une discipline stricto sensu.

\section{Conclusion: rapports entre les différentes didactiques}

Nous venons de voir que la didactique des mathématiques française a une ambition de construction théorique dont la portée dépasse le cadre disciplinaire, qu'il s'agisse d'un fait non assumé par la théorisation de départ ou au contraire qu'il s'agisse d'un cadre qui se revendique comme étant englobant. Il s'agit sans doute d'une spécificité à l'intérieur des didactiques disciplinaires qui, hors des mathé- 
matiques, même si elles se sont construites contre l'applicationnisme, reposent très souvent sur l'usage plus ou moins convertis de concepts théoriques issus de disciplines dites contributives. On remarque d'ailleurs que ce sont souvent des concepts issus de la didactique des mathématiques, comme celui de contrat didactique (Brousseau, 1986) ou de système didactique et de transposition didactique (Chevallard, 1985) qui sont souvent considérés comme des éléments d'un cadre théorique spécifiquement didactique (Bronckart \& Schneuwly, 1991; Schneuwly, 2001).

Selon les disciplines, selon les pays, un paradigme ou l'autre a été historiquement privilégié. Les raisons officielles de l'usage d'un paradigme ou d'un autre ressemblent parfois à des raisons épistémologiques, mais un examen attentif permet souvent de douter du fondement véritablement épistémologique (même s'il existe parfois, bien entendu). L'histoire des communautés de recherche, peu souvent mise en avant, pèse par contre très lourd sur les cadres utilisés, ce qui ne devrait pas à mon sens être refoulé. L'histoire collective et personnelle nous conduit en effet à être familiers voire spécialistes d'un paradigme ou d'un autre, qui devient alors le plus légitime, le plus naturel, voire le plus efficace, non parce qu'il est tel en théorie mais parce qu'il est tel dans la pratique de la recherche pour une communauté ou une personne donnée. Personne ne peut connaître à l'heure actuelle toutes les théories qui sont considérées comme légitimes concernant les phénomènes d'enseignement (même si l'on se restreint à une seule didactique disciplinaire!). Il devient alors très important de savoir quelle attitude un chercheur peut légitimement adopter vis-à-vis de paradigmes qui ne lui sont pas familiers.

Dans ma communauté de recherche d'origine, j'ai parfois eu le sentiment que le fait d'atteindre un résultat en s'appuyant sur des paradigmes différents conduisait à une sorte de lutte de légitimité entre ces paradigmes. Grosso modo, l'argument est le suivant: "puisque je peux atteindre ton résultat dans mon paradigme, je ne vois pas l'intérêt de l'obtenir autrement.» Je ne partage pas cette opinion, mais tout au contraire, celle de Georges Devereux (1972/1985):

[...] c'est précisément la possibilité d'expliquer "complètement» un phénomène humain d'au moins deux manières (complémentaires) qui démontre, d'une part, que le phénomène en question est à la fois réel et explicable, et, d'autre part, que chacune de ses deux explications est "complète» (et donc valable) dans son propre cadre de référence. (p. 13)

C'est parce qu'un même résultat peut être obtenu de plusieurs manières différentes que les paradigmes qui y conduisent sont confortés dans leur existence et qu'ils peuvent s'interroger sur leurs liens, voire leur complémentarité. Cela demande de s'interroger sur la notion de résultat. Si les concepts de didactique des mathématiques ne sont pas tous spécifiques des mathématiques et que certains ont une portée plus ample, par contre, il existe effectivement des résultats qui sont spécifiques des mathématiques, de l'algèbre, des équations du premier degré, etc. Si nous nous demandons qu'est-ce qu'un résultat en didactique des mathématiques, nous allons rencontrer autrement la spécificité. 
Un résultat en didactique est un bloc composé d'un cadre théorique explicite, et de données empiriques. Bien qu'une théorie ne soit jamais entièrement explicite, elle le sera plus en didactique qu'en physique, par exemple, à cause de l'absence de paradigme dominant;

A l'intérieur de ce bloc, il est nécessaire que le résultat résiste, qu'il soit stable. Il faut déjà bien sûr qu'il résiste aux faits dans des contextes semblables, similitude définie sous la responsabilité principale des chercheurs, ou plus de son cadre théorique. Et ce n'est pas une mince affaire;

Mais pour qu'il soit vraiment considéré comme un résultat, il faut qu'il puisse aussi se dégager notablement du cadre où il a été produit. Là, la compréhension de ce qu'on entend par cette exigence n'est pas évidente à cerner. Tant qu'on reste à l'intérieur du cadre théorique où la recherche a pris naissance, le problème est clair, même s'il n'est pas toujours simple. Mais il est inévitable de faire intervenir un facteur de légitimation pour fonder l'assertion que tel résultat est bien détachable.

En conséquence, la marque principale d'un résultat en didactique, c'est de renforcer le paradigme où il s'abrite.

[...] Finalement, la marque essentielle d'un résultat, c'est sa capacité à produire de nouveaux résultats. Soit par élimination, au moins temporaire, de questions que je n'ai plus besoin de me poser, soit par l'ouverture de nouvelles zones de recherches. (Johsua , 1996, pp. 214-215)

Ce que Johsua décrit ici, c'est un lien intime entre résultat et paradigme, tout résultat prenant un sens à l'intérieur d'un paradigme, ce qui ne permet pas de dépasser la question de la spécificité, mais qui cerne le lieu de validation d'un résultat.

Les évolutions récentes de la didactique des mathématiques et notamment la prise en compte des classes ordinaires et du rôle du professeur ont introduit la nécessité d'une autre réflexion concernant la généricité et la spécificité des théories didactiques, qui est maintenant une question ouverte dans les champs des didactiques, et plus largement dans celui des sciences de l'éducation.

Les relations entre les disciplines s'en trouvent ainsi bouleversées et il n'est alors pas étonnant de voir se développer de très nombreuses tentatives de croisement, de travail coopératif et même de comparaison entre les différentes didactiques disciplinaires (au sens de la didactique comparée, Mercier, SchubauerLeoni \& Sensevy, 2002; article de Schubauer-Leoni \& Leutenegger, dans ce volume). Les conséquences à la fois épistémologiques et institutionnelles de ce travail croisé ne sont pas encore stabilisées, l'avenir nous dira quelles solutions auront été retenues au plan de communautés ainsi rapprochées ou d'une communauté élargie. 


\section{Notes}

1 La première de ses institutions, créée en 1950, est la Commission Internationale pour l'Étude et l'Amélioration de l'Enseignement des Mathématiques (CIEAEM: voir pour un historique Keitel, Bazzini, Schopfer, Luelmo, Kraemer \& Inchley, 2002).

2 Structure complexe mise en place dans le cadre de l'IREM de Bordeaux, qui a fonctionné de 1972 à 1999 (voir Brousseau 1998 pour une description détaillée de ce dispositif).

3 Le moniteur de mathématiques, éditions Nathan, première édition en 1997.

4 Les paragraphes qui suivent (jusqu’à la définition des didactiques des savoirs) sont directement inspirés de cet article, pour ne pas alourdir le texte je ne le mentionne néanmoins pas systématiquement les pages correspondantes, sauf en cas de citation au sens strict du terme.

5 J'omets ici la formalisation qui demanderait une étude de l'article complet de Chevallard, 1992 (que je recommande au lecteur).

6 Comme en témoigne la conférence plénière de Anna Sfard au dernier International Congress for Mathematical Education, Copenhague, 2004 (disponible en DVD, commande sur le site web du congrès: http://www.icme-10.dk/index.html).

\section{Références bibliographiques}

Arsac, G. \& Mante, M. (1988). Le rôle du professeur: Aspects pratiques et théoriques, reproductibilité. Séminaire de didactique des mathématiques et de l'informatique, 101, 79-105

Artigue, M. (1988). Ingénierie didactique. Recherches en Didactique des Mathématiques, 9 (3), 281-308. Grenoble: La Pensée Sauvage.

Artigue, M., Gras, R., Laborde, C. \& Tavignot, P. (Ed.) (1994). Vingt ans de didactique des mathématiques en France. Grenoble: La Pensée Sauvage.

Bautier, E. \& Rochex, J.-Y. (2004). Activité conjointe ne signifie pas significations partagées. In C. Moro \& R. Rickenmann (Ed.), Situations éducatives et signification (Raisons Educatives, pp. 199-220). Bruxelles: De Boeck.

Bosch, M., Espinoza L. \& Gascón, J. (2003). El profesor como director de proceso de estudio: Anàlisis de organizaciones didàcticas espontàneas. Recherches en Didactique des Mathématiques, 23 (1), 79-196.

Bosch, M. \& Gascón, J. (2002). Organiser l'étude. Théories et empiries. In J.-L. Dorier, M. Artaud, M. Artigue, R. Berthelot \& R. Floris (Ed.), Actes de la 11ème Ecole d'Eté de Didactique des Mathématiques (pp. 23-40). Grenoble: La Pensée Sauvage.

Briand, J., Loubet, M. \& Salin M.-H. (2004). Apprentissages mathématiques en maternelle [CD-ROM]. Paris: Hatier.

Bronckart, J.-P. (2001). La psychologie ne peut être que sociale et la didactique est l'une de ses disciplines majeures. In J.-P. Bernié, Apprentissage, développement et significations (pp. 19419). Bordeaux: Presses universitaires de Bordeaux.

Bronckart, J.-P. \& Schneuwly, B. (1991). La didactique du français langue maternelle: l'émergence d'une utopie indispensable. Education et Recherche, 13, 8-26.

Brousseau, G. (1972). Processus de mathématisation. In La mathématique à l'Ecole Elémentaire (pp. 428-442). Paris: APMEP.

Brousseau, G. (1975). Exposé. Colloque L'analyse de la didactique des mathématiques. Bordeaux: IREM de Bordeaux.

Brousseau, G. (1981). Problèmes de didactique des décimaux: deuxième partie. Recherches en Didactique des Mathématiques, 2 (1), 37-127.

Brousseau, G. (1986). Fondements et méthodes de la didactique des mathématiques. Recherches en Didactique des Mathématiques, 7 (2), 33-115.

Brousseau, G. (1995). L'enseignant dans la théorie des situations didactiques. In R. Noirfalise (Ed.), Actes de la 8ème Ecole d'Eté de didactique des mathématiques (pp. 3-46). ClermontFerrand: IREM. 
Brousseau, G. (1998). Théorie des situations didactiques. Grenoble: La Pensée Sauvage.

Brun, J. (1994). Evolution des rapports entre la psychologie du développement cognitif et la didactique des mathématiques. In M. Artigue, R. Gras, C. Laborde \& P. Tavignot (Ed.), Vingt ans de didactique des mathématiques en France (pp. 51-66). Grenoble: La Pensée Sauvage.

Chevallard, Y. (1985). La transposition didactique. Grenoble: La Pensée Sauvage.

Chevallard, Y. (1992). Concepts fondamentaux de la didactique: perspectives apportées par une approche anthropologique. Recherches en Didactique des Mathématiques, 12 (1), 73 111.

Chevallard, Y. (1997). Familière et problématique, la figure du professeur. Recherches en Didactique des Mathématiques, 17 (1),17-54.

Chevallard, Y. (2002a). Organiser l'étude. Structures et fonctions. In J.-L. Dorier, M. Artaud, M. Artigue, R. Berthelot \&. R. Floris (Ed.), Actes de la 11ème Ecole d'Eté de Didactique des Mathématiques (pp. 3-22). Grenoble: La Pensée Sauvage.

Chevallard, Y. (2002b). Organiser l'étude. Ecologie et régulation. In J.-L. Dorier, M. Artaud, M. Artigue, R. Berthelot \& R. Floris (Ed.), Actes de la 1 lème Ecole d'Eté de Didactique des Mathématiques (pp. 41-56). Grenoble: La Pensée Sauvage.

Chevallard, Y., Bosch, M. \& Gascón, J. (1996). Estudiar matemáticas, el eslabón perdido entre enseñanza y aprendizaje. Barcelona: I.C.E. \& Horsori.

Comiti, C., Grenier, D. \& Margolinas, C. (1995). Niveaux de connaissances en jeu lors d'interactions en situation de classe et modélisation de phénomènes didactiques. In G. Arsac, J. Gréa, D. Grenier \& A. Tiberghien (Ed.), Différents types de savoirs et leur articulation (pp. 92-113). Grenoble: La Pensée Sauvage.

Conne, F. (1992). Savoir et connaissance dans la perspective de la transposition didactique. Recherches en Didactique des Mathématiques, 12 (2-3), 221-270.

Devereux, G. (1985). Ethnopsychanalyse complémentariste. Paris: Flammarion. (Traduction française de l'original en anglais publié 1972)

Goigoux, R. (sous presse). Ressources et contraintes dans le travail d'enseignement de la lecture au cours préparatoire. In B. Schneuwly \& Th. Thévenaz (Ed.), Le travail de l'enseignant et l'objet enseigné: le cas du français langue première. Bruxelles: De Boeck Université.

Johsua , S. (1996). Qu'est-ce qu'un «résultat» en didactique des mathématiques. Recherches en Didactique des Mathématiques, 16 (2), 197-220.

Keitel, C. Bazzini, L. Schopfer, E. Luelmo, M.-J. Kraemer, J.-M. \& Inchley C. (2002). La Commission internationale pour l'étude et l'amélioration de l'enseignement des mathématiques est le plus ancien groupe internationale de travail et de réflexion de l'éspecialité d'éducation mathématique [sic!], [http://www.upc.es/info/cieaem54/cieaem-fra/cieaem-presen.htm].

Loquet, M., Garnier, A. \& Amade-Escot, Ch. (2002). Transmission des savoirs en activités physiques, sportives et artistiques dans des institutions différentes: enseignement scolaire, entraînement sportif, transmission chorégraphiques. Revue Française de Pédagogie, 141, 99-109. Paris: INRP.

Margolinas, C. (1992a). Eléments pour l'analyse du rôle du maître: les phases de conclusion. Recherches en Didactique des Mathématiques, 12 (1), 113-158.

Margolinas, C. (1992b). Analyse de situation et analyse du rôle du maître sur un cas particulier. Séminaire de didactique des mathématiques et de l'informatique, 138, 185-205.

Margolinas, C. (1993). De l'importance du vrai et le faux dans la classe de mathématiques. Grenoble: La pensée sauvage.

Margolinas, C. (1999). Les pratiques de l'enseignant: Une étude de didactique des mathématiques: recherche de synthèses et perspectives. In M. Bailleul (Ed.), Actes de la 10ème Ecole d'Eté de Didactique des Mathématiques (pp. 10-33). Caen: IUFM de Caen et A.R.D.M.

Margolinas, C. (2000). La production des faits en didactique des mathématiques. Actes du séminaire du LIREST (pp. 33-55). Cachan: E.N.S. 
Margolinas, C. (2002). Situations, milieux, connaissances - analyse de l'activité du professeur. In J.-L. Dorier, M. Artaud, M. Artigue, R. Berthelot \& R. Floris (Ed.), Actes de la 11ème Ecole d'Eté de Didactique des Mathématiques (pp. 141-156). Grenoble: La Pensée Sauvage.

Margolinas, C. (2004). Points de vue de l'élève et du professeur: Essai de développement de la théorie des situations didactiques. Habilitation à diriger les recherches en sciences de l'éducation, Université de Provence.

Margolinas, C. (2005). La dévolution et le travail du professeur. In P. Clanché, M.-H. Salin \& B. Sarrazy (Ed.), Autour de la théorie des situations (pp. 329-333). Grenoble: La pensée sauvage.

Margolinas, C. (sous presse). Les situations à bifurcations multiples: indices de dysfonctionnement ou de cohérence. In A. Mercier \& C. Margolinas (Coord.), Balises en didactique des mathématiques [CD-ROM]. Grenoble: La Pensée Sauvage.

Margolinas, C. \& Goigoux, R. (sous presse). Une comparaison de l'activité d'une maîtresse de cours préparatoire en français et en mathématiques. In A. Mercier \& C. Margolinas (Coord.), Balises en didactique des mathématiques [CD-ROM]. Grenoble: La Pensée Sauvage.

Margolinas, C. \& Thomazet S. (2004). Dévolution différenciée en classe de CP. Manuscrit soumis pour publication.

Mercier, A. (1998). La participation des élèves à l'enseignement. Recherches en Didactique des Mathématiques, 18 (3), 279-310.

Mercier, A., Schubauer-Leoni, M.-L. \& Sensevy, G. (2002). Vers une didactique comparée. Revue Française de Pédagogie, 141, 5-16, Paris: INRP.

Perrin-Glorian, M.-J. (1994). Théorie des situations didactiques: naissance, développements, perspectives. In M. Artigue, R. Gras, C. Laborde \& P. Tavignot (Ed.), Vingt ans de didactique des mathématiques en France (pp. 97-147). Grenoble: La Pensée Sauvage.

Robert, A. (2001). Les recherches sur les pratiques des enseignants et les contraintes de l'exercice du métier d'enseignant. Recherches en Didactique des Mathématiques, 21 (1-2), 221270.

Roditi, E. (2005). Les pratiques enseignantes en mathématiques: Entre contraintes et liberté pédagogiques. Paris: L'Harmattan.

Rogalski, J. (2003). Y a-t-il un pilote dans la classe ? Une analyse de l'activité de l'enseignant comme gestion d'un environnement dynamique ouvert. Recherches en Didactique des $\mathrm{Ma}$ thématiques, 23 (3), 343-388.

Schneuwly, B. (2001). Les concepts de la didactique du français: rareté et foisonnement. In M. Marquilló Larruy (Ed.), Questions d'épistémologie en didactique du français (pp. 46-50). Poitiers: Les Cahiers FORELL et Université de Poitiers.

Vergnaud, G. (1981). L'enfant, la mathématique et la réalité. Berne: Peter Lang.

Vergnaud, G. (1991). La théorie des champs conceptuels. Recherches en didactique des mathématiques, $10(2 / 3), 133-169$.

Vergnaud, G. (1994a). Apprentissages et didactiques, où en est-on ? Paris: Hachette.

Vergnaud, G. (1994b). Le rôle de l'enseignant à la lumière des concepts de schème et de champ conceptuel. In M. Artigue, R. Gras, C. Laborde \& P. Tavignot (Ed), Vingt ans de didactique des mathématiques en France (pp. 177-191). Grenoble: La Pensée Sauvage.

Vergnaud, G. (2005). Repères pour une théorie psychologique de la connaissance. In A. Mercier \& C. Margolinas (Coord.), Balises en didactique des mathématiques (pp. 123-136). Grenoble: La Pensée Sauvage.

Mots clés: didactique des mathématiques, théorie des situations, théorie des champs conceptuels, théorie anthropologique du didactique 


\section{Versuch einer Genealogie der Mathematikdidaktik}

\section{Zusammenfassung}

Das Anliegen dieses Artikels ist es, die Genealogie des Forschungsgebiets der Mathematik $\neg$ didaktik in Frankreich allgemein verständlicher zu machen. Zu diesem Zweck beziehe ich die Brüche, die erst zu diesem Forschungsgebiet geführt haben, mit ein und vergleiche sie mit den Grundsteinen anderer Fachdidaktiken. Danach zeige ich das Besondere der Gründungstheorien der Mathematikdidaktik auf: die Theorie der didaktischen Situationen, die Theorie der Konzeptionsfelder und die anthropologische Theorie der Didaktik. Schließlich betrachte ich eine neuere Entwicklungsphase, die geprägt ist durch den Einbezug von gewöhnlichen Klassen und durch eine Veränderung in der Lehrerrolle. Schliesslich zeige ich auf, wie die Mathematikdidaktik mit ihrem anfänglich eher engen fachspezifischen Konzept zu einer treibenden Kraft im intensiven Austausch mit anderen Fachdidaktiken und anderen Teilen der Erziehungswissenschaft wurde.

Schlagworte: Mathematikdidaktik, Theorie der didaktischen Situationen, Theorie der Konzeptionsfelder, anthropologische Theorie der Didaktik

\section{Saggio sulla genealogia in didattica della matematica}

\section{Riassunto}

Questo articolo cerca di fornire delle chiavi per una migliore comprensione della genealogia del campo della ricerca in didattica della matematica in Francia. Nella prima parte considero le rotture che sono all'origine di questo campo di ricerca ed evidenzio le differenze e le somiglianze con l'evoluzione di altre didattiche. Ciò permetterà di mettere a fuoco le specificità delle teorie fondatrici della didattica della matematica: la teoria delle situazione didattiche, la teoria dei campi concettuali, la teoria antropologica della didattica. Di seguito passo a descrivere una fase più recente caratterizzato dalla considerazione delle classi "ordinarie" e del ruolo dell'insegnante. In conclusione mostrerò come la didattica della matematica sia passata da un'identità disciplinare molto delimitata ad un'apertura con interazioni intense con altre didattiche e altri campi delle scienze dell'educazione.

Parole chiave: Didattica della matematica, teoria delle situazioni, teoria dei campi concettuali, teoria antropologica del didattico 


\title{
A Genealogy of Mathematics Didactics
}

\begin{abstract}
The article at hand aims at a better understanding of the genealogy of research in mathematics didactics in France. It takes a look at the disruptions that actually created this field of research, and compares it with the starting point of other didactics. Then follows a display of the particularities of the main theories of mathematics didactics in France: The theory of didactical situations, the theory of conceptual fields, and the anthropological theory of didactics. Finally, a recent developmental phase, characterized by taking into account 'ordinary' classes and changes in the teachers' position, is described. In conclusion, the intention is to show how mathematics didactics have evolved from a rather narrow branch-specific concept to a driving force of important interactions with other didactics and fields of educational science.
\end{abstract}

Key words: Mathematics didactics, theory of situations, theory of conceptual fields, anthropological theory of didactics 